\title{
A case report of unacknowledged hypercalcemia in emergency
}

\author{
Granieri $L^{1}$, Ferrazza $P^{2 *}$, Capria $F^{2}$, Ariello $M^{1}$, Zibella F $^{1}$, CastaldoR ${ }^{1}$ and Del Gaudio Salvatore ${ }^{1}$ \\ ${ }^{1}$ Department of Emergency Medicine, Pineta Grande Hospital, Castel Volturno, Caserta, Italy \\ ${ }^{2}$ Centro Ricerca LNAge, Pineta Grande Hospital, Castel Volturno, Caserta, Italy
}

\begin{abstract}
Hypercalcaemia is a common laboratory finding which may be due either to hyperparathyroidism or to paraneoplastic syndrome. Its symptoms may include asthenia, decreased intestinal motility, electrocardiographic abnormalities, nephrocalcinosis or deposition of calcium salts in other tissues such as gallbladder, myocardium and blood vessels. Neurologic complications may develop into alteration of consciousness, hallucination, convulsions, somnolence and coma. Calcium homeostasis may be influenced by that of other cations such as magnesium and kalium, whose regulation mechanisms are stricltly related with the calcium ones. In this article, the case of a patient with unacknowledged hypercalcemia who developed severe complications and coma following the start of magnesium sulphate therapy for the treatment of ventricular tachycardia, is presented. In emergency, unacknowledged hypercalcemia may represent a serious risk for the patient therefore a timely assessment of calcium is recommended in order to avoid potentially fatal complications.
\end{abstract}

\section{Introduction}

Hypercalcaemia, an increase of the calcium plasma levels, is a common laboratory finding which in $90 \%$ of the cases is due either to hyperparathyroidism or to paraneoplastic syndrome [1].

The symptoms of hypercalcaemia (Table 1) are non-specific and depend on the severity of the event as well as on the rapidity of its occurrence [2]. They commonly include asthenia, decreased intestinal motility, electrocardiographic abnormalities such as a shortened QT interval associated with increased sensitivity to digitalis. Nephrocalcinosis or, occasionally, deposition of calcium salts in other tissues such as gallbladder, myocardium and blood vessels may represent a common finding as well [3].

Table 1. Hypercalcemia symptoms and signs.

\begin{tabular}{|l|l|}
\hline Central Nervous System & Lethargy \\
\hline & Depression \\
\hline & Stupor \\
\hline Neuromuscular & Come \\
\hline & Asthenia \\
\hline & Proximal Myopathy \\
\hline Cardiovascular & Muscular Hypotrophy \\
\hline & Hypertension \\
\hline & Bradycardia \\
\hline Renal & Enhancement of digitalis toxicity \\
\hline & Polyuria \\
\hline & Nephrolithiasis \\
\hline Gastro-intestinal & Nephrocalcinosis \\
\hline & Nausea, vomit \\
\hline & Constipation \\
\hline & Dyspepsia \\
\hline & Peptica ulcer \\
\hline & Pancreatitis \\
\hline Soft tissue calcinosis & Itch \\
\hline & Chondrocalcinosis \\
\hline & Nephrocalcinosis \\
\hline
\end{tabular}

Although unusually, the clinical presentation of hypercalcemia may include neurologic disturbances such as headache and anorexia associated with dizziness and vomit. In most serious cases neurologic complications may develop into alteration of consciousness with the occurrence of hallucination, convulsions, somnolence and finally coma $[4,5]$. Calcium homeostasis, regulated by several mechanisms including the binding of the mineral to plasma proteins, the intracellular $\mathrm{pH}$ and the active uptake of the ion mediated by ATP-dependent pumps and the cellular emission through $\mathrm{Na}^{+} / \mathrm{Ca}^{2+}$ exchangers located on the cellular membranes, may be influenced by that of other cations such as magnesium and kalium, whose regulation mechanisms are stricltly related with the calcium ones [6,7].

In this article we present the case of a patient with unacknowledged hypercalcemia, who developed severe complications and coma following the start of magnesium sulphate therapy for the treatment of ventricular tachycardia.

\section{Case report}

Patient of male gender, aged 76 and weighed $75,0 \mathrm{~kg}$ presented to the emergency room with marked asthaenia, minimal effort dyspnea associated with difficulty walking and gastric dyspepsia.

Vital signs at admission were the following: heart rate (HR) 105 bpm, with atrial fibrillation detected by a cardiac monitor; oxygen saturation $\left(\mathrm{SpO}_{2}\right)$ 89\% on room air; blood pressure 110/60 mmh; normal body temperature $\left(36.3^{\circ} \mathrm{C}\right)$; and glycemia $105 \mathrm{mg} / \mathrm{dL}$. Physical examination resulted in: orthopnea; normal peripheral vascular exam; massive edema on both lower extremities and the sacrum; harsh vesicular murmur, lower in the inferior region and in the right-sided middle-chest area, with crackles in the left-sided middle-chest area;

Correspondence to: Paolo Ferrazza, L N Age Clinical Trial Center, Pineta Grande Hospital, Castel Volturno, Italy, E-mail: paolo.ferrazza@lnage.it

Received: June 29, 2017; Accepted: July 25, 2017; Published: July 27, 2017 
anxiety. The abdomen was apparently treatable, even though a thorough inspection could not be carried out since the patient had to maintain the orthopneic position.

Patient's anamnesis was significant for cardiac disorders, presenting history of congestive heart failure (CHF) due to dilated, post-ischemic cardiomyopathy associated with serious reduction of the systolic function $(<20 \%)$. The patient was a pacemaker/defibrillator carrier and had already undergone percutaneous transluminal coronary angioplasty (PTCA) twice, with three drug-eluting stents (DES). Furthermore, among several hospitalizations for cardiac insufficiency, 4 events had happened within the previous 8 months.

A chest X-ray (resulted in bilateral hilar congestion and pleural effusion on the right side), an electrocardiogram (ECG) (resulted in paced rhythm) and blood withdrawal were immediately performed at the emergency room before transfer to the medical unit of the emergency department. Laboratory tests revealed the following significant abnormalities: increase of creatinine levels $(2,1 \mathrm{mg} / \mathrm{dL}$; reference ranges $0,8-1,2 \mathrm{mg} / \mathrm{dL})$; azotaemia $(184 \mathrm{mg} / \mathrm{dL} ; \mathrm{RR} 10-40$ $\mathrm{mg} / \mathrm{dL}$ ); increased alkaline phosphatase (ALP) $(578 \mathrm{mU} / \mathrm{ml} ; \mathrm{RR}$ 80-306 mU/ml); and increased reactive C Protein (RCP) (15 mg/L; RR 0-1 mg/L). Blood count, sodium, potassium, cardiac enzymes, lipid profile and thyroid hormones were all normal. According with the hospital routine, at admission the calcium levels were not investigated. The following pharmacological treatment was started: subcutaneous enoxaparin (4000 IU/day) and oral administration of furosemide (50 $\mathrm{mg}$ twice a day), pantoprazole (one $20 \mathrm{mg}$ tablet/day), acetylsalicylic acid (ASA) (100 mg/day), atorvastatin $(20 \mathrm{mg} /$ day $)$, enalapril $(2,5 \mathrm{mg}$ twice a day) and bisoprolol (2,5 $\mathrm{mg}$ twice a day). On the third day the overall patient's clinical picture showed reduction of the edema as well as of the dyspnea and improvement in renal function. A telemetry revealed the presence of polymorphic ventricular tachycardia hence the following treatment was added to the abovementioned pharmacological therapy: intravenous infusion of magnesium solphate ( 2 gr. in 10 minutes); amiodarone (one $200 \mathrm{mg}$ tablet three times/ day); continuous intravenous infusion $(25 \mathrm{~mL} / \mathrm{h})$ of saline $(500 \mathrm{~mL}$ of $0,9 \%$ sodium chloride) with the addition of potassium chloride (60 $\mathrm{mEq})$ and magnesium sulphate ( $3 \mathrm{gr})$. The morning after the patient was found comatose, with a Glasgow Coma Scale (GCS) score of 9 (E2 V2 M5). The patient was immediately submitted to the following investigations: head computerized tomography (CT) scan (negative for acute disease), ammonemia (negative) and calcemia (abnormal: $16,8 \mathrm{mg} / \mathrm{dL}$; RR $8,0-11,8 \mathrm{mg} / \mathrm{dL}$ ). Oral administration of bisoprolol and furosemide was immediately stopped, the speed of intravenous infusion was increased up to $200 \mathrm{~mL} / \mathrm{h}$, and the overall therapy was completed with the addition of the following: dobutamine $(5 \mathrm{mcg} /$ $\mathrm{kg} / \mathrm{h})$, dopamine $(6 \mathrm{mcg} / \mathrm{kg} / \mathrm{h})$, furosemide $(2 \mathrm{mg} / \mathrm{h})$, clodronic acid (300 mg/day) and desametasone (4 mg/day). After 72 hours' treatment with this regimen the patient, still comatose (GCS score of 9), showed haemodynamic stability, improved renal function and the following relevant biochemistry results: creatininemia $1,5 \mathrm{mg} / \mathrm{dL}$; azotaemia 74 $\mathrm{mg} / \mathrm{dL}$; normal kalium $(3,6 \mathrm{mg} / \mathrm{dL}$; RR 3,5- $5 \mathrm{mg} / \mathrm{dL})$ and magnesium $(2,1 \mathrm{mg} / \mathrm{dL} ; \mathrm{RR} 1,7-2,2 \mathrm{mg} / \mathrm{dL}$ ) levels. Despite a proper diuresis (about $6 \mathrm{Lt} /$ day), calcium levels were still high $(14,8 \mathrm{mg} / \mathrm{dL})$ and associated with hypophosphoraemia ( $1.8 \mathrm{mg} / \mathrm{dL}$; RR $2,5-4 \mathrm{mg} / \mathrm{dL}$ ), low 25-hydroxy vitamin D3 (28,1 ng/mL; RR 30-80 ng/mL) and increased parathyroid hormone (PTH) levels (1.067 pg/ml; RR 11-67 pg/mL). The infusion of magnesium sulphate was then discontinued and, within 24 hours, patient's neurological conditions clearly improved, calcemia gradually decreased in parallel with the occurrence of hypomagnesemia $(1,2 \mathrm{mg} /$ $\mathrm{dL}$; RR 1,7 - 2,2 mg/dL).

Fourteen days later, the general clinical picture was stabilized and the patient was discharged with mild hypercalcemia $(13 \mathrm{mg} / \mathrm{dL})$ and hypomagnesemia $(1,6 \mathrm{mg} / \mathrm{dL})$. The diagnosis of hyperparathyroidism was accompanied by the recommendation for further investigation and the referral to endocrinology specialists.

\section{Discussion}

Hypercalcemia, a marked elevation of serum calcium levels, if not adequately treated is a potentially fatal condition characterized by a combination of symptoms and signs whose combination may be configured as "hypercalcemic syndrome", whose seriousness depends both on the severity and on the time over which the calcium level increase develops [2,3].

Although serious, both in severe and acute cases neurological symptoms of hypercalcemia are often unrecognized and can be attributable to the membrane-stabilizing effect of calcium, since its increase may entail a reduction of either cellular and neuromuscular excitability, commonly associated with asthenia and fatigue. These symptoms are reversible as long as the medical treatment is started early [8]. The calcium ion also modulates the activity of diverse enzymes and plays a critical role in several physiologic processes such as the release of pituitary hormones, catecholamines and neurotransmitters. Due to the ion's importance, the homeostasis of calcium is finely regulated and its serum concentration may even be influenced by other ions, such as kalium and magnesium, whose control machinery is intimately related the calcium's one. In particular, magnesium plays a crucial role in the maintenance of the resting membrane potential of both nerve and muscle cells, as well as in the transmission of the nerve impulse, and it facilitates the production and secretion of the parathyroid hormone (PTH) $[6,7,9]$. Indeed, although the effects of slight fluctuations of magnesium levels on PTH secretion are still unclear, it has been observed that extreme variations of the ion's concentration out of the ranges of normality may dramatically influence PTH secretion: cronic hypomagnesemia in fact, is associated with a reduction in PTH secretion and therefore with a functional hypoparathyroidism and hypocalcemia [10], which may be due to an essential role played by ionized magnesium in the process of PTH release carried out by the parathyroid secretory granules. On the reverse side, the effects of acute variations of magnesium levels out of the ranges of normality are qualitatively similar to those of the calcium ion: PTH secretion is inhibited by hypermagnesemia and induced by hypomagnesemia [11]. In the reported case, the administration of magnesium sulphate for the treatment of polymorphic ventricular tachycardia in a patient most likely suffering from unacknowledged hypercalcemia and hypomagnesemia resulted in a dramatic worsening of the hypercalcemic crisis: the correction of magnesium leves in fact, might have induced the physiologic effects of PTH furtherly exacerbating hypercalcemia. The hypomagnesemia induced by the administration of furosemide and by the interruption of magnesium supplementation might have induced a functional block of PTH and reduced the PTH-calcium feedback loop.

\section{Conclusions}

In the presented case, the main peculiarity consisted in the seriousness and rapidity of the clinical manifestations of hypercalcemia occurred after the administration of magnesium sulphate, as well as in the uneffective response to the standard hypercalcemia medical treatment, apparently refractory to the suspension of magnesium infusion. The general principles of the treatment for acute hypercalcemia do not depend on its etiology: in acute phase the appropriate medical intervention should immediately be implemented while the etiology appraisal aimed at verifying whether the hypercalcemia is $\mathrm{PTH}$-dependent or not (Figure 1) should be postponed, in order to 


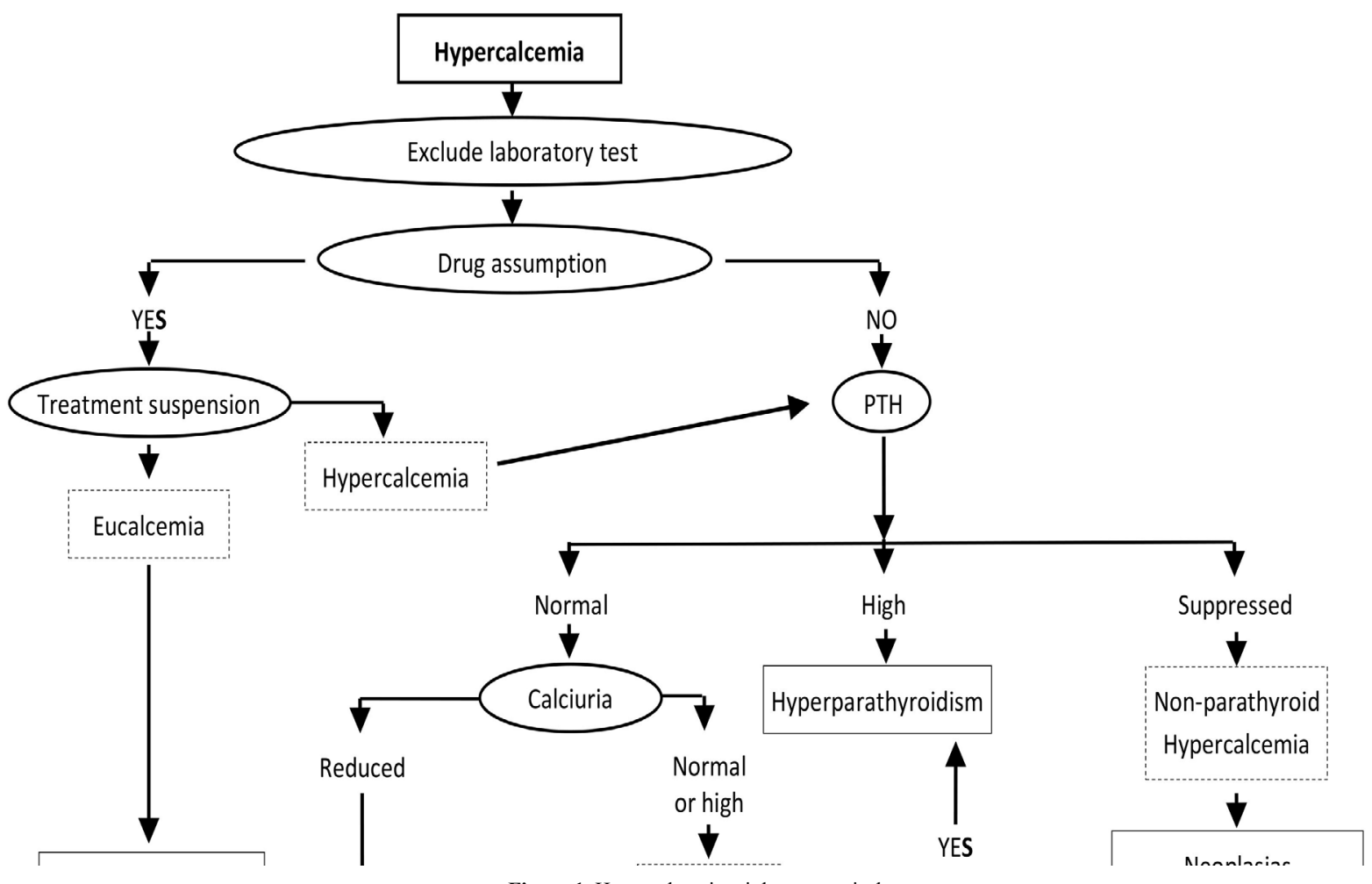

Figure 1. Hypercalcemia etiology appraisal

establish the proper diagnostic and therapeutic plan only once the life-threatening situation has been definitely brought under control. In emergency, following the administration of magnesium sulphate, either for the control of acute seizures in cases of serious gravidic toxemia or for the treatment of ventricular arrhytmia and torsades de pointes (TdP), it would be appropriate to proceeed with the assessment of calcemia every time, in order to prevent the risk of coma induced by an unacknowledged or paucisymptomatic hypercalcemic state. Moreover, in cases of serious, standard-treatment-refractory, potentially fatal hypercalcemia, the induction of marked hypomagnesemia associated with rigorous cardiac monitoring should be considered.

\section{References}

1. Zofkova I (2016) Hypercalcemia: pathophysiological aspects. Physiol Res 65: 1-10. [Crossref]

2. Assadi F (2009) Hypercalcemia: An Evidence-Based Approach to Clinical Cases. Iran J Kidney Dis 3: 71-79. [Crossref]

3. Ziegler R (2001) Hypercalcaemic crisis. J Am Soc Nephrol 12: S3-S9. [Crossref]
4. Kearney T, Dang C (2007) Diabetic and endocrine emergencies. Postgrad Med J 83:79-86. [Crossref]

5. Gurrado A, Piccinni G, Lissidini G, Di Fronzo P, Vittore F, et al. (2012) Hypercalcaemic crisis due to primary hyperparathyroidism - a systematic literature review and case report. Endokrynol Pol 63: 494-502. [Crossref]

6. Felsenfeld A, Rodriguez M, Levine B (2013) New insights in regulation of calcium homeostasis. Curr Opin Nephrol Hypertens 22: 371-376. [Crossref]

7. Blaine J, Chonchol M, Levi Renal M (2015) Renal Control of Calcium, Phosphate, and Magnesium Homeostasis Clin J Am Soc Nephrol 10: 1257-1272. [Crossref]

8. Walker J (2015) Diagnosis and management of patients with hypercalcaemia. Nurs Older People 27: 22-26. [Crossref]

9. Grober U (2015) Magnesium in prevention and therapy. Nutrients 7: 8199-8226. [Crossref]

10. Pironi L, Malucelli E, Guidetti M, Lanzoni E, Farruggia G, et al. (2009) The complex relationship between magnesium and serum parathyroid hormone: a study in patients with chronic intestinal failure. Magnesium Research 22: 37-43. [Crossref]

11. Paunier L (1992) Effect of magnesium on phosphorus and calcium metabolism. Monatsschr Kinderheilkd 140: S17-20. [Crossref] 